\title{
LOSS OF MAGNETIC FLUX AND ANGULAR MOMENTUM FROM MOLECULAR CLOUDS
}

\author{
Takenori Nakano \\ Nobeyama Radio Observatory, National Astronomical Observatory \\ Nobeyama, Minamisaku, Nagano 384-13, Japan
}

\section{Introduction}

The magnetic field and the angular momentum are major obstacles against cloud contraction. I will review recent results on magnetic flux loss rate and angular momentum loss rate and will investigate a gross feature of cloud contraction.

\section{Magnetic Flux Loss}

\subsection{MAGNETIC FLUX PROBLEMS}

In an oblate cloud penetrated by magnetic fields parallel to its minor axis, the mean magnetic force is weaker than the self-gravitational force only when its magnetic flux $\Phi$ is less than a critical flux

$$
\Phi_{\mathrm{cr}}^{(\mathrm{f})} \approx 2 \pi G^{1 / 2} M
$$

where $G$ is the gravitational constant and $M$ is the cloud mass (e.g., Strittmatter 1966). Thus, a cloud with $\Phi>\Phi_{\mathrm{cr}}^{(\mathrm{f})}$ must lose some of its initial flux before it can begin dynamical contraction (for more accurate criterion, see $\S 4$ ). Moreover, the magnetic flux to mass ratio for an interstellar cloud is estimated to be several $10^{2}$ to $10^{5}$ times greater than the ratio for a magnetic star (Nakano 1983, 1984). Therefore, clouds, or more exactly, cloud cores, must lose most of their initial flux at some stage of star formation.

\subsection{PROCESSES OF MAGNETIC FLUX LOSS}

As is well known, ohmic dissipation is quite inefficient in ordinary clouds. Mestel and Spitzer (1956) pointed out that in a slightly ionized cloud the magnetic field and ions, which are frozen to the magnetic field, drift in a sea of neutral gas, and magnetic flux decreases gradually. Elmegreen (1979) investigated the effect of charged grains, which may be only weakly coupled to the magnetic field, on magnetic flux loss. At very high densities even ions are not frozen to field lines. The transition from strong coupling to weak coupling of particles with magnetic fields occurs gradually, and the density at which this transition effectively occurs depends sensitively on the kind of particles, being especially influenced by the particle mass. Since various kinds of charged particles are contained in a cloud and their relative abundances change 
greatly with density (see $\$ 2.4$ below), the study of magnetic field dissipation requires careful treatment. Nakano (1984) and Nakano and Umebayashi (1986a) introduced a formalism with which magnetic field dissipation can be treated quite generally.

\subsection{CLOUDS WITH REALISTIC GRAIN SIZE DISTRIBUTION}

With this formalism Nakano (1984), Nakano and Umebayashi (1986a, b), and Umebayashi and Nakano (1990) investigated magnetic field dissipation assuming that all grains have the same radius, typically $a=0.1 \mu \mathrm{m}$. In reality, however, interstellar grains have a wide size distribution. For example, Mathis, Rumpl, and Nordsieck (1977, referred to as MRN hereafter) deduced a power law size distribution for graphite and silicate grains,

$$
\frac{d n_{\mathrm{gr}}}{d a}=A n_{\mathrm{H}} a^{-3.5}, \quad a_{\min }<a<a_{\max }
$$

where $n_{\mathrm{H}}$ is the gas density by hydrogen number, $a_{\min } \lesssim 100 \AA, a_{\max } \approx 2500 \AA$, and $A \approx 1.5 \times 10^{-25} \mathrm{~cm}^{2.5}$ (Draine and Lee 1984; Mathis 1986). In addition, recent obscrvations suggest the existence of very small "grains" with $a$ as small as $3 \AA$ (Sellgren 1984; Leger and Puget 1984; Draine and Anderson 1985).

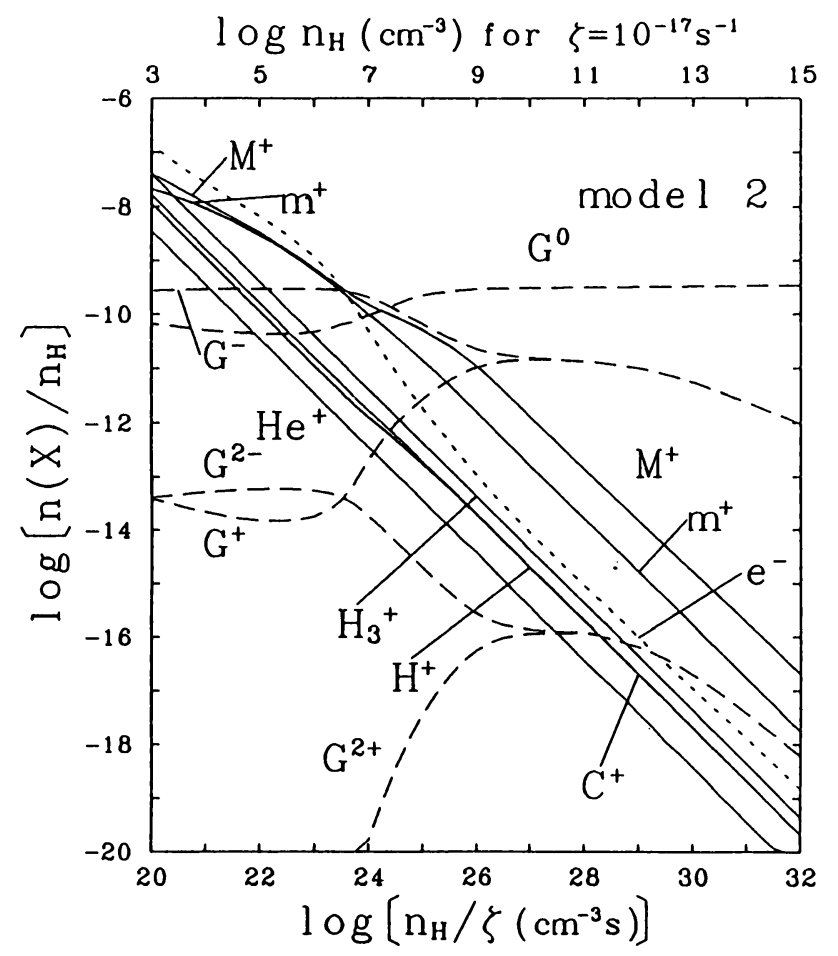

Figure 1. Abundances of various charged particles in clouds with ice-mantled grains (model 2) as functions of the density, $n_{\mathrm{H}}$. Grains are denoted as $\mathrm{G}$ with a superscript representing their electric charge. 
Tiny grains have two effects on magnetic field dissipation. First, the large total surface area of tiny grains allows efficient recombination of ions and electrons on grain surfaces. This suppresses the ion density in clouds and has an effect of enhancing field dissipation. Second, most grains are electrically charged, and smaller grains couple more strongly with magnetic fields. This has the effect of suppressing field dissipation. Since both effects are large but operate in opposing directions, a detailed investigation is needed in order to determine which effect is dominant and how efficient field dissipation can be. The following is a summary of the recent work by Nishi, Nakano, and Umebayashi (1990).

We adopt the following four grain models: (1) the standard MRN distribution $(50 \AA<a<2500 \AA) ;(2)$ the MRN distribution with ice-mantles $(90 \AA<a<4500 \AA)$; (3) the extended MRN distribution without ice-mantles $(3 \AA<a<2500 \AA)$; (4) the standard MRN distribution plus an additional population of grains of size $a=4 \AA$ with number density $2 \times 10^{-7} n_{\mathrm{H}}$. Although a particle as small as $3-4 \AA$ may have properties quite different from ordinary grains, we neglect the effects due to such properties. Models 3 and 4 should be regarded as extreme cases for the effects of tiny grains mentioned above.

\subsection{DENSITIES OF CHARGED PARTICLES}

In clouds shielded from ultraviolet radiation, ions are first formed by ionization of hydrogen molecules and helium atoms by cosmic rays. Many of molecular ions, denoted as $\mathrm{m}^{+}$, recombine with electrons dissociatively. But some of them undergo charge-transfer reactions with metal atoms, forming metal ions, denoted as $\mathbf{M}^{+}$. Metal ions recombine radiatively with electrons. All ions can recombine with electrons which have been adsorbed on grain surfaces. This reaction scheme is nearly in steady state. Figure 1 shows steady state abundances of various charged particles for model 2 (ice-mantled grains). In this model, molecular ions and metal ions are dominant charged particles at densities $n_{\mathrm{H}} \lesssim 10^{7} \mathrm{~cm}^{-3}$. At higher densities, however, charged grains are more abundant than ions.

\subsection{MAGNETIC FLUX LOSS FROM CLOUD CORES}

Quasistatic contraction of a cloud due to magnetic flux loss is highly nonhomologous. Nakano (1979) showed that a high-density core appears, which subsequently contracts much faster than the other parts of the cloud. The timescale of such nonhomologous contraction is nearly equal to the timescale of magnetic flux loss from the core whose size across field lines is nearly equal to the size along field lines. Figure 2 shows the timescale, $t_{B}$, of magnetic flux loss from cloud cores as a function of the core density, $n_{\mathrm{H}}$, for each of the four grain models. We have assumed that the magnetic flux, $\Phi$, of the core is nearly equal to the critical flux $\Phi_{\mathrm{cr}}^{(\mathrm{f})}$ given by equation (1). The flux loss time, $t_{B}$, is 10 to $10^{2}$ times the free-fall time, $t_{\mathrm{f}}$, at densities lower than a critical density $n_{\mathrm{cr}}^{(1)}$. For model $2, n_{\mathrm{cr}}^{(1)} \approx 10^{9} \mathrm{~cm}^{-3}$, for example. The flux loss time is smaller than $t_{\mathrm{f}}$ only at densities higher than another critical density $n_{\mathrm{cr}}^{(2)}$. For model $2, n_{\mathrm{cr}}^{(2)} \approx 10^{10} \mathrm{~cm}^{-3}$. Since a cloud cannot contract faster than free fall, the magnetic field is decoupled from the gas at $n_{\mathrm{H}} \gtrless n_{\mathrm{cr}}^{(2)}$. We find that charged grains are more efficient than ions in preventing the drift of magnetic fields at densities $n_{\mathrm{H}} Z 2 \times 10^{4} \mathrm{~cm}^{-3}$ for model 2 . Thus, even at ordinary densities of molecular clouds, grains are important in preventing magnetic flux loss.

When $\Phi<\Phi_{\mathrm{cr}}^{(\mathrm{f})}, t_{B}$ is proportional to $\Phi^{-2}$ at $n_{\mathrm{H}} \ll n_{\mathrm{cr}}^{(1)}$, and is almost indepen- 
dent of $\Phi$ at $n_{\mathrm{H}} \gtrsim n_{\mathrm{cr}}^{(2)}$. In the latter density range, ohmic dissipation is dominant.

Since a cloud with $\Phi<\Phi_{\mathrm{cr}}^{(\mathrm{f})}$ contracts dynamically, or nearly freely, assuming that the cloud mass is much greater than the Jeans critical mass, and also $t_{B} \gg t_{\mathrm{f}}$ at $n_{\mathrm{H}} \lesssim n_{\mathrm{cr}}^{(1)}$, flux loss down to far below $\Phi_{\mathrm{cr}}^{(\mathrm{f})}$ does not occur at densities $n_{\mathrm{H}} \lesssim 10^{9} \mathrm{~cm}^{-3}$ for any of the four grain models.

\subsection{FRAGMENTATION OF CLOUDS DUE TO MAGNETIC FLUX REDISTRIBUTION}

As shown in $\S 2.5$, the flux loss time, $t_{B}$, is a decreasing function of the density, $n_{\mathrm{H}}$. This means that a part of a cloud with higher density loses magnetic flux faster, and then contracts faster, than a part with lower density, assuming that the high-density part has a mass exceeding the Jeans critical mass. Therefore, a density fluctuation in a cloud is amplified by magnetic flux redistribution, and the cloud breaks into fragments. This process was first pointed out by Nakano (1976) using his old results, and also works with our new results.

\section{Angular Momentum Loss}

The most efficient process of removing cloud's angular momentum is transport of it to an ambient medium via magnetic torque. Magnetic braking of cloud's rotation has been investigated for some cases of magnetic configuration around a cloud.

\subsection{CLOUDS WITH UNIFORM MAGNETIC FIELD}

The first investigation of magnetic braking was for a cloud penetrated by a uniform

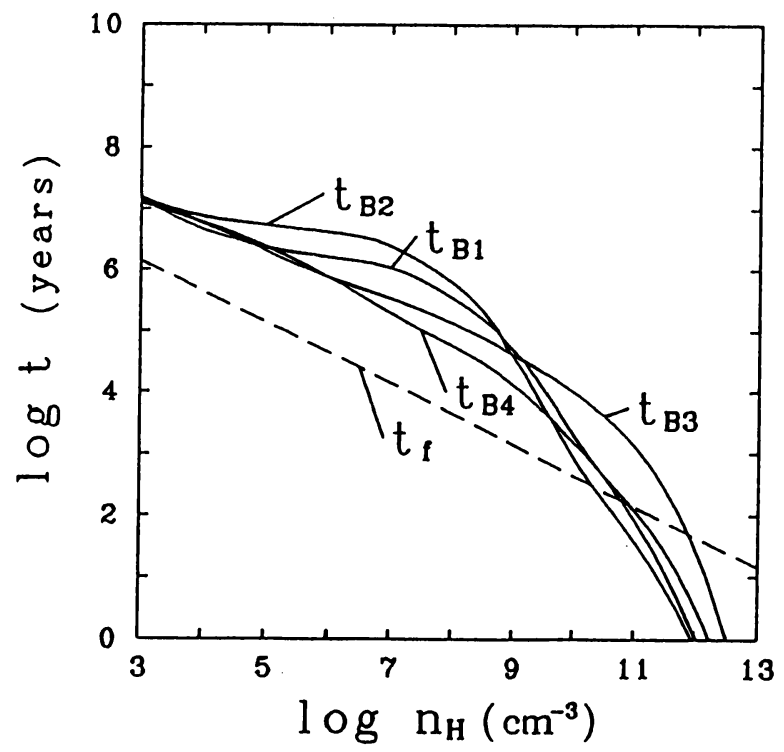

Figure 2. The magnetic flux loss time for cloud cores in which magnetic force nearly balances the self-gravitational force as a function of the core density, $n_{\mathrm{H}}$, for each of four grain models. 
magnetic field with a rotation axis parallel to field lines. The angular momentum of such a cloud is lost in a time taken by a torsional Alfvén wave to cross a domain in the ambient medium whose column density is equal to the column density of the cloud (Ebert et al. 1960; Mouschovias and Paleologou 1980). However, such a magnetic configuration is realized only around a cloud with strong magnetic field $\left(\Phi \gg \Phi_{\mathrm{cr}}^{(\mathrm{f})}\right)$, or with weak gravity, where field configuration is hardly affected by cloud's gravity. Stars hardly form in such a cloud.

\subsection{CONTRACTED CLOUDS}

The magnetic field around a cloud with strong gravity is distorted considerably. Gillis, Mestel, and Paris $(1974,1979)$ investigated angular momentum loss from such a cloud assuming that the poloidal component of magnetic field is fixed. However, the assumed poloidal field is not force-free, and magnetic configuration changes in an Alfvén-crossing time, which is usually much shorter than the spin-down time of the cloud. These pioneering works are insufficient in this sense.

\subsection{CLOUD CORES}

As mentioned above, contraction of a magnetized cloud is highly nonhomologous and only a core finally contracts to form a star. Therefore, angular momentum loss from a cloud core is essential in star formation. Let us consider an axisymmetric cloud with a core at the center. Although the magnetic field may be nearly uniform very far from the cloud, there is an intermediate zone where field lines anchored to the core are nearly radial and decline only slightly from the symmetry axis. We can take the field in this zone nearly force-free. Assuming that the intermediate zone is sufficiently large, Nakano (1989) investigated the transport of core's angular momentum to this zone. The density in the intermediate zone is assumed to be proportional to $r^{-n}$, where $r$ is the distance from the cloud center. It is convenient to introduce $\nu \equiv 1 /(6-n)$. The results are as follows.

For $\nu=1 / 2(n=4)$, propagation of torsional Alfvén waves can be solved analytically, and the rotation velocity of the core, $\Omega_{\mathrm{cc}}$, is given by

$$
\Omega_{\mathrm{cc}}(\tau)=\Omega_{\mathrm{i}} \exp \left(-s_{1 / 2} \tau\right)
$$

where $\tau$ is a dimensionless time, $\Omega_{\mathrm{i}}$ is the initial rotation velocity of the core, and $s_{\nu}$ is a quantity determined by the ratio of the density just outside the core and the mean core density.

For $\nu$ between 0 and $1 / 2(n<4)$, the solution is obtained semianalytically as

$$
\Omega_{\mathrm{cc}}(\tau) \approx \frac{\Omega_{\mathrm{i}}}{1-\nu} \exp \left(s_{\nu} \tau \cos \frac{\pi}{2-2 \nu}\right) \cos \left(s_{\nu} \tau \sin \frac{\pi}{2-2 \nu}\right)-\frac{\Omega_{\mathrm{i}} \nu /(1-\nu)}{\left(1+a_{\nu} s_{\nu} \tau\right)^{2-2 \nu}}
$$

where $a_{\nu}$ is a constant of order unity. The first term oscillates with time with the amplitude decreasing exponentially. The second term decreases with time only by a power law and finally prevails over the first term.

In all cases most of the initial angular momentum is lost in a timescale equal to $s_{\nu}^{-1}$. This is the time taken by a torsional Alfvén wave to cross a domain whose moment of inertia is equal to the moment of inertia of the cloud core.

\subsection{DISTRIBUTION OF ROTATION VELOCITY IN CLOUDS}

In cases other than $n=4$, spin-down of a core is rather complicated. The $n=4$ case is special; the Alfvén velocity is constant along field lines, and the moment of inertia 
of the gas in a magnetic tube with unit length is also constant along field lines. This is why waves propagate smoothly and the core loses angular momentum smoothly. In other cases wave propagation is rather complicated. Reflection of waves occurs everywhere. This is why the core behaves in a complicated way.

The second term of equation (4) represents a rotation reverse to the initial rotation. Because a core has a much smaller moment of inertia than the outer part of the cloud, the core loses angular momentum faster than the outer part. Therefore, after a few spin-down timescales, the core rotates reversely to the outer part, though this retrograde rotation gradually decays afterwards. Thus, there must be a stage in which a core rotates reversely to the outer part. With minute observations we may be able to find clouds with such rotational structure.

\section{Contraction of Rotating Magnetized Clouds}

A gross feature of contraction of rotating magnetized clouds can be obtained by comparing the contraction time with the magnetic flux loss time and with the angular momentum loss time, as done by Nakano (1990). The following is a summary of the results.

First, let us consider a cloud core whose initial magnetic flux, $\Phi_{i}$, is greater than a critical flux, $\Phi_{\mathrm{cr}}^{(\mathrm{d})}$, defined by equation (6) just below. The cloud is embedded in a medium which has uniform magnetic field and uniform density far from the cloud. The timescale of quasistatic contraction of the core with flux $\Phi$ is given by

$$
t_{\mathrm{cont}} \equiv\left(\frac{d \ln R_{\mathrm{cc}}}{d t}\right)^{-1} \approx \frac{1}{2}\left[1-\left(\frac{\Phi_{\mathrm{cr}}^{(\mathrm{f})}}{\Phi}\right)^{2}\right] t_{B},
$$

where $R_{\mathrm{cc}}$ is an equilibrium radius of the cloud core, which we determine from the virial theorem, and $t_{B}$ is the magnetic flux loss time investigated in $\S 2$. Contraction can be regarded quasistatic only when $t_{\text {cont }}>t_{\mathrm{f}}$, or $\Phi$ is greater than

$$
\Phi_{\mathrm{cr}}^{(\mathrm{d})} \approx \Phi_{\mathrm{cr}}^{(\mathrm{f})}\left(1+\frac{t_{\mathrm{f}}}{t_{B}}\right) .
$$

When $\Phi$ decreases to $\Phi_{\mathrm{cr}}^{(\mathrm{d})}$, dynamical contraction sets in as long as the core mass, $M_{\mathrm{cc}}$, is considerably greater than the Jeans critical mass, $M_{\mathrm{J}}$.

An outline of contraction is shown in Figure 3. In an early stage of a core with $\Phi_{\mathrm{i}}>\Phi_{\mathrm{cr}}^{(\mathrm{d})}$, the angular momentum loss time, $t_{L}$, is much smaller than $t_{\text {cont }}$, and so the core loses angular momentum, $L$, rapidly, and soon settles down in corotation with the ambient medium. After this, contraction is induced by magnetic flux loss, and $L$ decreases with contraction keeping corotation with the ambient medium because $t_{L}<t_{\text {cont }}$. As contraction proceeds, the ratio $t_{L} / t_{\text {cont }}$ increases. When the Alfvén velocity, $V_{\mathrm{A}}^{(0)}$, in the ambient medium is greater than the sound velocity, $V_{\mathbf{s}}$, in the core, a condition $t_{L}>t_{\text {cont }}$ is realized and angular-momentum-conserving contraction sets in while the core is still contracting quasistatically. Afterwards, $\Phi$ decreases to $\Phi_{\mathrm{cr}}^{(\mathrm{d})}$, and dynamical contraction sets in. When $V_{\mathrm{A}}^{(0)} \lesssim V_{\mathrm{s}}$, dynamical contraction begins while the core is still in corotation with the ambient medium. Afterwards, the core shifts to angular-momentum-conserving contraction.

A cloud with $\Phi_{\mathrm{i}}$ slightly smaller than $\Phi_{\mathrm{cr}}^{(\mathrm{d})}$ contracts dynamically from the beginning losing angular momentum because $t_{L}$ is nearly equal to the magnetically diluted free-fall time, $t_{\mathrm{f}}^{\prime}$, assuming the cloud mass $M \gg M_{\mathrm{J}}$. However, it is unclear whether corotation with an ambient medium is realized or not. 


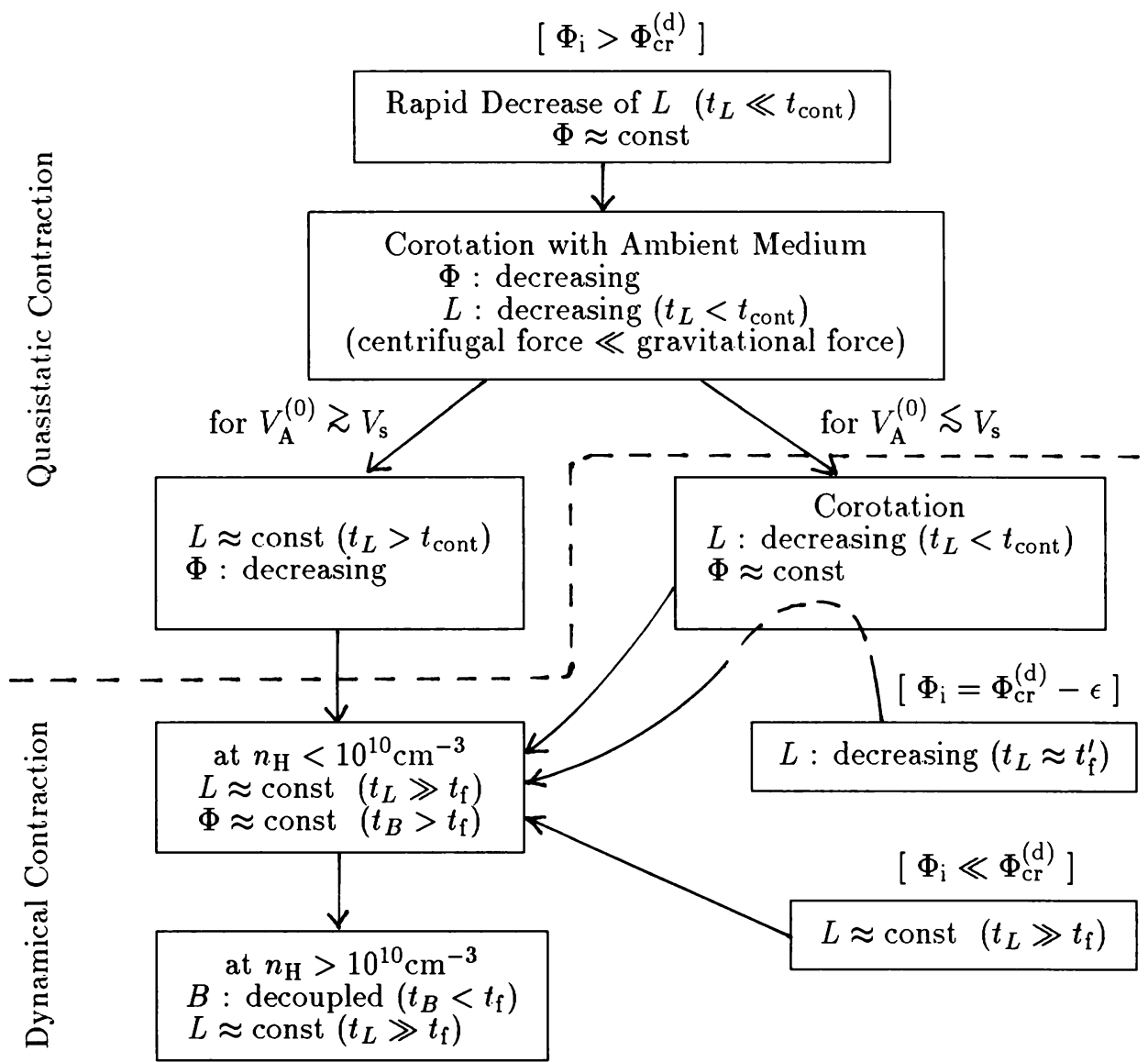

Figure 3. Gross feature of contraction of rotating magnetized clouds. The dashed line corresponds to the stage of magnetic flux $\Phi=\Phi_{\mathrm{cr}}^{(\mathrm{d})} \approx \Phi_{\mathrm{cr}}^{(\mathrm{f})}\left(1+t_{\mathrm{f}} / t_{B}\right)$. In stages above this line the clouds contract quasistatically, and below this line they contract dynamically.

A cloud with $\Phi_{\mathrm{i}} \ll \Phi_{\mathrm{cr}}^{(\mathrm{d})}$ contracts dynamically from the beginning, as long as $M>M_{\mathrm{J}}$, nearly conserving $L$.

In dynamically contracting clouds, magnetic flux $\Phi$ is nearly conserved at $n_{\mathrm{H}}$ less than the critical density $n_{\mathrm{cr}}^{(2)}$, which was obtained in $\S 2$ and is $10^{10} \mathrm{~cm}^{-3}$ for model 2 (ice-mantled grains). At $n_{\mathrm{H}} \gtrsim n_{\mathrm{cr}}^{(2)}$ the magnetic field is decoupled from the neutral gas and $\Phi$ decreases rapidly. Angular momentum is conserved in this stage.

Let us go back to the case of $\Phi_{\mathrm{i}}>\Phi_{\mathrm{cr}}^{(\mathrm{d})}$ with $V_{\mathrm{A}}^{(0)} \gtrless V_{\mathrm{s}}$. The angular-momentumconserving contraction effectively sets in at a stage $t_{L} \approx t_{\text {cont }}$, and angular momentum of a core is fixed at this stage. If binary stars form from this core, we can 
predict the orbital period of the binary stars. Assuming that stars of equal mass are formed, Nakano (1990) found that the orbital period is

$$
P_{\mathrm{b}} \approx 3 \mathrm{yr}\left(\frac{\Omega_{0}}{2 \cdot 10^{-15} \mathrm{rad} \mathrm{s}^{-1}}\right)^{3}\left(\frac{10^{5} \mathrm{~cm}^{-3}}{n_{L}}\right)^{2} \approx 3 \mathrm{yr}\left(\frac{\Omega_{0}}{2 \cdot 10^{-15} \mathrm{~s}^{-1}}\right)^{3}\left(\frac{10^{2} \mathrm{~cm}^{-3}}{n_{0}}\right)^{2},
$$

where $n_{L}$ is the core density at the stage $t_{L} \approx t_{\text {cont }}$ and is about $10^{3}$ times the density, $n_{0}$, of the ambient medium, and $\Omega_{0}$ is the rotation velocity of the ambient medium. If we take $n_{0}$ as a mean density of a molecular cloud complex whose rotation velocity $\Omega_{0}$ is nearly equal to the shear of galactic rotation, we obtain observed orbital periods of binary stars. Thus, the magnetic flux loss rate and the angular momentum loss rate shown above are consistent with orbital periods of binary stars.

\section{References}

Draine, B. T., and Anderson, N., 1985. Astrophys. J., 292, 494.

Draine, B. T., and Lee, H. M., 1984. Astrophys. J., 285, 89.

Ebert, R., Hoerner, S. von \& Temesvary, S., 1960. Die Entstehung von Sternen durch Kondensation diffuser Materie, p. 315, Springer-Verlag, Berlin.

Elmegreen, B. G., 1979. Astrophys. J., 232, 729.

Gillis, J., Mestel, L. \& Paris, R. B., 1974. Astrophys. Space Sci., 27, 167.

Gillis, J., Mestel, L. \& Paris, R. B., 1979. Mon. Not. Roy. Astr. Soc., 187, 311.

Leger, A., and Puget, J. L., 1984. Astr. Astrophys., 137, L5.

Mathis, J. S., 1986. Astrophys. J., 308, 281.

Mathis, J. S., Rumpl, W., and Nordsieck, K. H., 1977. Astrophys. J., 217, 425.

Mestel, L. \& Spitzer, L., Jr., 1956. Mon. Not. Roy. Astr. Soc., 116, 503.

Mouschovias, T. Ch. \& Paleologou, E. V., 1980. Astrophys. J., 237, 877.

Nakano, T., 1976. Publ. Astr. Soc. Japan, 28, 355.

Nakano, T., 1979. Publ. Astr. Soc. Japan, 31, 697.

Nakano, T., 1983. Publ. Astr. Soc. Japan, 35, 209.

Nakano, T., 1984. Fund. Cosmic Phys., 9, 139.

Nakano, T., 1989. Mon. Not. Roy. Astr. Soc., 241, 495.

Nakano, T., 1990. Mon. Not. Roy. Astr. Soc., 242, 535.

Nakano, T. \& Umebayashi, T., 1986a. Mon. Not. Roy. Astr. Soc., 218, 663.

Nakano, T. \& Umebayashi, T., 1986b. Mon. Not. Roy. Astr. Soc., 221, 319.

Nishi, R., Nakano, T., and Umebayashi, T., 1990. submitted to Astrophys. J. Sellgren, K., 1984. Astrophys. J., 277, 623.

Strittmatter, P. A., 1966. Mon. Not. Roy. Astr. Soc., 132, 359.

Umebayashi, T., and Nakano, T., 1990. Mon. Not. Roy. Astr. Soc., 243, 103. 